\title{
ATTRACTORS FOR SECOND ORDER NONAUTONOMOUS LATTICE SYSTEM WITH DISPERSIVE TERM
}

\author{
XiaOlin Xiang — Shengfan Zhou
}

\begin{abstract}
In this paper, we prove the existence of pullback attractor, pullback exponential attractor and uniform attractor for second order nonautonomous lattice system with dispersive term and time-dependent forces. Then we prove the existence of uniform exponential attractor for the system driven by quasi-periodic external forces.
\end{abstract}

\section{Introduction}

It is known that there are two important tools to study the asymptotic behavior of non-autonomous evolution equations (see [10], [6], [15]). The first one is the uniform attractor by introducing a so-called skew-product semiflows on a larger phase space, which allows one to embed a given non-autonomous system into an autonomous semiflow, and then to appeal to the general theory of autonomous semiflows. The second one is the pullback attractor (or kernel sections) directly for non-autonomous equations. The uniform attractor and pullback attractor are natural generalizations of the notion of global attractor for a non-autonomous dynamical system. However, these two attractors are usually infinite dimensional and sometimes attract orbits at a relatively slow

2010 Mathematics Subject Classification. 60H15, 34D35, 35Q80.

Key words and phrases. Second order lattice system, dispersive term, pullback attractor, pullback exponential attractor, uniform attractor, uniform exponential attractor.

This work is supported by the National Natural Science Foundation of China under Grant No. 11471290, 11071165, 61271396, Zhejiang Natural Science Foundation under Grant No. LY14A010012 and Zhejiang Normal University Foundation under Grant No. ZC304014012. 
speed leading to take an unexpected long time to be reached, thus the uniform exponential attractor and the pullback exponential attractor having finite fractal dimension and attracting all bounded sets exponentially were introduced, and they have become appropriate alternatives to study the asymptotic behavior of non-autonomous dynamical systems.

Recently, there are several works about the existence of uniform attractor, pullback attractor, kernel sections, pullback and uniform exponential attractor for non-autonomous lattice dynamical systems (LDSs), which arise in many applied areas, see [1]-[4], [9], [17], [21], [22], [24], and the references therein. Of those works, Zhou and Han in [21] and [22] presented some sufficient conditions for the existence of pullback and uniform exponential attractor for the continuous process on Banach space and space of infinite sequences, and applied them to prove the existence of pullback exponential attractors for the first order and partly dissipative non-autonomous LDSs and uniform exponential attractors for the non-autonomous Klein-Gordon-Schrödinger and Zakharov LDSs. Motivated by [21], [22], in this article, we consider the pullback attractor, pullback exponential attractor, uniform attractor for the following second order non-autonomous lattice dynamical system with dispersive term and time-dependent forces

$$
\ddot{u}_{i}+\beta(A \ddot{u})_{i}+\alpha \dot{u}_{i}+\gamma(A \dot{u})_{i}+\lambda u_{i}+(A u)_{i}+f_{i}\left(u_{i}\right)=g_{i}(t),
$$

and the uniform exponential attractor for second order non-autonomous lattice system driven by quasi-periodic external forces:

$$
\ddot{u}_{i}+\beta(A \ddot{u})_{i}+\alpha \dot{u}_{i}+\gamma(A \dot{u})_{i}+\lambda u_{i}+(A u)_{i}+f_{i}\left(u_{i}\right)=a_{i} h_{i}(\widetilde{\sigma}(t)),
$$

where $i \in \mathbb{Z}, t \geq \tau, \tau \in \mathbb{R}, u=\left(u_{i}\right)_{i \in \mathbb{Z}}, u_{i} \in \mathbb{R}, \beta \geq 0, \gamma>0, \alpha>0, \lambda>0$, $a_{i} \in \mathbb{R}, g_{i} \in C(\mathbb{R}, \mathbb{R}), h_{i}, f_{i} \in C^{1}(\mathbb{R}, \mathbb{R}), \tilde{\sigma}(t) \in \mathbf{T}^{n}$ (n-dimensional torus), $A$ is a non-negative and self-adjoint linear operator with the decomposition $A=$ $\bar{D} D=D \bar{D}$, and $D$ is defined by

$$
(D u)_{i}=\sum_{l=-m_{0}}^{l=m_{0}} d_{l} u_{i+l}, \quad\left|d_{l}\right| \leq c_{0}, \quad \text { for all } u=\left(u_{i}\right)_{i \in \mathbb{Z}}, \quad-m_{0} \leq l \leq m_{0},
$$

and $\bar{D}$ is the adjoint of $D$. If $A$ is defined by $(A u)_{i}=2 u_{i}-u_{i-1}-u_{i+1}$, then (1.1) can be regarded as a discrete analogue of the following continuous fourth order partial differential equation in $\mathbb{R}$ :

$$
u_{t t}-\beta u_{x x t t}+\alpha u_{t}-\gamma u_{x x t}+\lambda u-u_{x x}+f(u, x)=g(x, t),
$$

which is a mathematical model for describing the spread of longitudinal strain waves in nonlinear elastic rods and weakly nonlinear ion-acoustic waves; see, e.g. [5], [11] and the references therein. The terms $-\beta u_{x x t t}$ and $-\gamma u_{x x t}$ are called the dispersive and the viscosity dissipative terms, respectively. In the autonomous case (i.e. $g$ is independent of $t$ ) defined in a bounded domain and the stochastic 